\title{
Micro RNA-499C Induces The Differentiation of Mouse Embryonic Stem Cells (mESCs) Into Cardiomyocytes
}

\section{Abstract}

Background: A microRNA, miR499c, has been discovered in human fetal heart which rescues mutant hearts in cardiac nonfunction mutant axolotl (salamander) embryos and also induces the differentiation of mouse embryonic stem cells (mESCs) to form into definitive cardiomyocytes.

Results: Eight days after transfection with MiR499c, approximately $75-80 \%$ of the stem cells develop typical cardiomyocyte morphologies and express the cardiac specific marker, Troponin $T$, as well as the muscle proteins, tropomyosin and $\alpha$-actinin, as shown by immunohistochemical staining. qRT-PCR confirms that transfection with MiR499c increases expression of troponin $T$ and tropomyosin and further shows an increased expression of myosin as well as Wnt11 and Sox17. Untreated controls do not show significant expression of these proteins.

Conclusion: It is evident that the miR499c induces the development of contractile myofibrils characteristic of striated cardiac muscle indicating that the miR499c microRNA plays an important role in the differentiation of cardiomyocytes.

\section{Keywords}

Micro RNA-499c, myofibrillogenesis, cardiac troponin T, mouse embryonic stem cells (mESCs)
Andrei Kochegarov, Ashley Arms, Michael C. Hanna, Larry F Lemanski ${ }^{1}$

1 Department of Biological and Environmental Sciences, Texas A\&M University-Commerce, PO Box 3011, Commerce, TX 75429-3011 2, USA.

\section{Contact information:}

Dr. Larry F. Lemanski.

Distinguished Research Professor and Head, Department of Biological and Environmental Sciences, Texas A\&M University-Commerce, Commerce, Texas 75429-3011 USA

巨Larry.Lemanski@tamuc.edu

\section{Background}

MicroRNAs (miRs) are an important class of small non-protein-encoding RNAs that function in transcriptional and post-transcriptional re- 
gulation of gene expression including regulation of cardiac development, cardiomyocyte proliferation and differentiation. They act to control the expression of sets of genes and fundamental regulatory pathways and are thought of as master regulators of gene expression. In a recent publication, Eulalio et al. showed that transduction of microRNAs in mouse hearts increases both DNA synthesis and induces cell proliferation [1]. In our recent publication we have reported the discovery of a novel form of microRNA, miR-499c, which rescues mutant hearts in salamander embryos [2]. The role of miR-499 in cardiomyocyte differentiation has been highlighted by several recent publications and it has been shown that miR-499 expression is significantly increased in differentiated cardiomyocytes [3]. Expression of miR-499 in human cardiac stem cells represses the miR-499 target genes, Sox6 and Rod1, enhancing cardiomyogenesis in vitro. Also, expression of miR499 is significantly increased after myocardial infarction, suggesting that expression of miR-499 may provide an important protective mechanism against heart injury [3]. Another study demonstrated that miR-499 promotes the differentiation of mesenchymal stem cells into cardiomyocytes [4]. Thus, these data suggest that miR-499 plays a central role in cardiac development and differentiation.

\section{Methods}

\section{RNA cloning}

Random clones of 396 individual RNAs from human fetal heart were successfully acquired using the pDONR222 plasmid as a vector by routine procedures we have described earlier [2]. DNA clones were synthesized by PCR using the vectors as templates with M13 primers and the PCR products were visualized by agarose gel electrophoresis after ethidium bromide staining. The RNAs were synthesized by using an in vitro transcription reaction. RNA clones along with $0.1 \mathrm{mg} / \mathrm{ml}$ of lipofectamine transfection reagent were diluted to a concentration of $7 \mathrm{ng} / \mu \mathrm{l}$ in Opi-Mem media and transfected into the stem cells.

\section{Stem cell culture protocol}

Mouse Embryonic Stem Cells (mESCs, Strain 129, OriCell, Cyagen Biosciences, Inc., Santa Clara, CA) were incubated and grown on coverslips at $37^{\circ} \mathrm{C}$ and $5 \% \mathrm{CO}$, and passaged routinely according to our published protocols [2]. To induce differentiation, the mouse cells were transfected with RNA derived from the active clones that had been mixed with the transfection reagent, Lipofectamine RNAiMAX (Life Technologies, Grand Island, NY), diluted to a concentration of $50 \mathrm{ng} / \mu \mathrm{l}$ in OPTI-MEM medium (Life Technologies, Grand Island, NY) and incubated for 6 hours.

\section{qRT-PCR}

Using a NucleoSpin RNAll Kit (Macherey-nagel, Bethlehem, PA), RNA was extracted from differentiated cells treated with the active RNA and controls were either untreated or treated only with lipofectamine. qRT-PCR was performed with a Rotor-Gene machine using a Rotor-Gene SYBR PCR kit (Qiagen, Valencia, CA) with primers designed as described in our earlier paper, Kochegarov et al [2].

\section{Immunohistochemistry}

Cells grown on coverslips were fixed in 4\% paraformaldehyde for $30 \mathrm{~min}$, rinsed in PBS with 3\% BSA for 3 min, permeabilized in $0.1 \%$ Tween-20 and 3\% BSA and stained overnight at $4{ }^{\circ} \mathrm{C}$ with primary antibodies including: 1. monoclonal anti-tropomyosin CG3 antibody (Developmental Studies Hybridoma Bank, University of lowa, lowa City, IA) diluted to 1:75; 2. anti-cardiac troponin antibodies ( $T$ 564766, BD Pharmingen, San Jose, CA), diluted to 1:100; 3. Anti- $\alpha$-actinin antibodies (Developmental Studies Hybridoma Bank, University of lowa, lowa City, IA) diluted 1:100. The cells on the coverslips were rinsed with PBS containing 3\% BSA for 3 min and stained with FITC-labelled anti-mouse antibodies ((Abcam, 
Cambridge, MA) diluted to $1: 75$ for $2 \mathrm{hr}$ at $4^{\circ} \mathrm{C}$. The coverslips with the attached cells were mounted on glass microscope slides with SlowFade $®$ Gold antifade reagent (Invitrogen, Grand Island, NY) and viewed under an Olympus Fluorview laser scanning confocal microscope at an excitation of $488 \mathrm{~nm}$ and emission of $520 \mathrm{~nm}$ for FITC. The images were recorded using a computerized digital camera linked to the microscope.

\section{Results}

Mouse ESCs were cultured during multiple passages as undifferentiated colonies on matrigel (BD Biosciences, San Jose, CA) (Fig. 1A). The passaged stem cells were placed in small "hanging drops" on Petri dish lids and incubated for 48 hours. After 48 hours, most of the stem cells had aggregated and formed embryoid bodies (EBs) (Fig. 1B). We plated these EBs on collagen-coated culture dishes and allowed them to attach, grow and proliferate for an additional 48 hours (Fig.1C). At this stage we treated the stem cells with RNA derived from the active miR-499c clone. After 8 days in culture most of the cells (75-80\%) differentiated into cells with characteristic cardiomyocyte morphologies (Fig.1D) and these cells showed significant staining for tropomyosin and cardiac specific troponin $T$ and $\alpha$-actinin (Figs. 1E, 3B, 4). The quantity of cells that exhibited spontaneous cardiomyocyte differentiation without RNA treatment was less than $10 \%$ after 8 days in culture.

We also tested the ability of miR-499c to induce differentiation directly without the formation of EBs. We treated mouse embryonic stem cells daily for 6 hours with transfection medium containing the miR-499c followed by normal growth medium. The mouse embryonic stem cells differentiated into cardiomyocytes within 8 days showing changes into cardiomyocyte type morphologies and expressing cardiac troponin $\mathrm{T}$ in $75-80 \%$ of the cells. When the fluorescent staining intensity for tropomyosin was measured in the differentiating stem cells and converted into digital values by computer analysis, during the first seven days in culture the cellular tropomyosin increased progressively, strongly su-

Figure 1: Timeline showing different stages in myocardiogenesis of mouse ESCS. A. This colony of mouse ESCS shows a distinct margin indicative of a relatively undifferentiated colony of ESC; $B$. Embryoid body has formed from cells in hanging drop cultures; C. Spreading and proliferation of cells on gelatin coated tissue culture plates; D. Phase contrast image of mouse ESCs treated with cardiogenic microRNA-499c. A majority of the cells have taken on shapes characteristic of early stage myocardiocytes; E. Differentiated cells after 8 days of treatment with the cardiogenic inducing microRNA-499c stained with antibody against tropomyosin. The cells show significant accumulations of tropomyosin suggesting a myocardial phenotype..

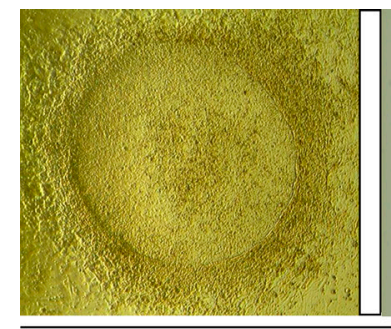

A

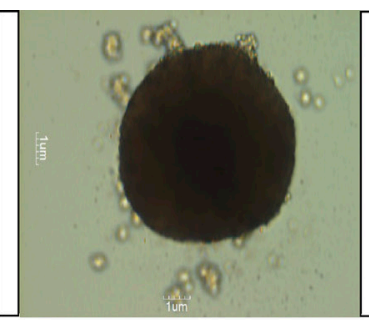

B

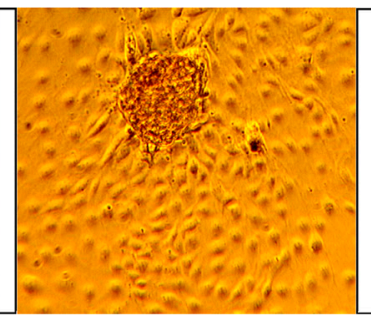

C

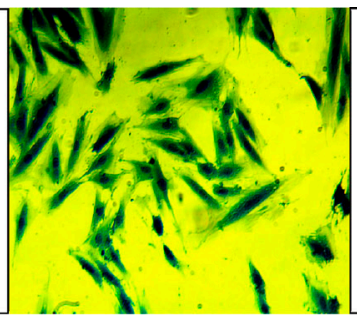

D

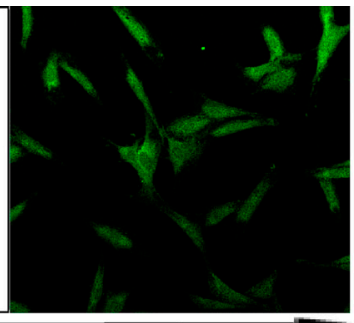

E 
ggesting that the differentiation of the stem cells into cardiomycytes was taking place (Fig. 2).

Also after 7 days of treatment with mir499c, the stem-cells acquired characteristic cardiomyocyte morphologies (Fig. 3A) and showed tropomyosin (Figs. 3B, 4B), troponin $T$ (Fig. 4A) and $\alpha$-actinin (Fig. 4C) incorporated into myofibrils indicative of definitive cardiomyocytes (Figs. 3B, 4). In untreated controls, most of the stem cells became fibroblastlike in appearance without significant tropomyosin or troponin expression (Figs. 3C, 3D). High resolution confocal imaging revealed myofibril organization after immunofluorecent staining for the cardiac specific troponin $\mathrm{T}$, tropomyosin and $\alpha$-actinin

Figure 2: Time course of stem cell-derived cardiomyocyte differentiation revealed by immunofluorescent staining with antibodies for tropomyosin after treatment with microRNA-499c. The fluorescently labeled cells are scanned for brightness and intensity and quantitated by computer digitization. The data show a gradual increase of the tropomyosin expression up to 7 days. Numbers on the bottom of the graph indicate day(s) after the microRNA-499 transfection of the stem cells. Numbers on the side represent the relative intensities of the fluorescent staining in the microscope fields with the brightest field ( 7 days) being assigned a value of 100 .

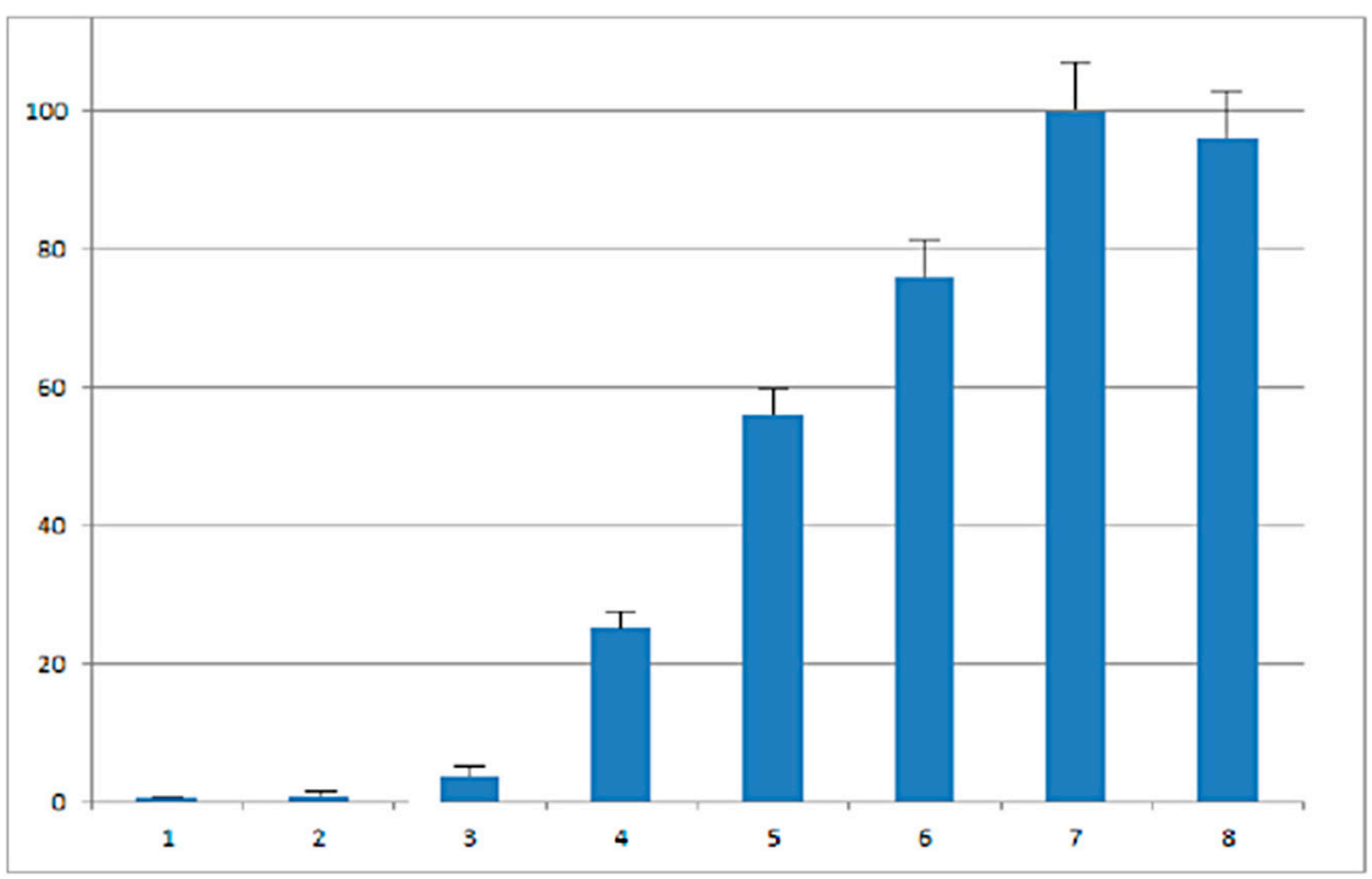


Figure 3: Mouse ESCs differentiated into spindle-shaped cardiomyocytes 8 days after transfection with microRNA-499 (A, B); mouse ESC 8-day parallel controls, without transfection (C, D). A. Many of the microRNA-499c-treated stem cells show characteristic cardiomyocyte shapes; $B$. The treated cells stain intensely for tropomyosin with anti-tropomyosin antibodies; C. Untreated cells have shapes that are more typical of fibroblasts and other non-cardiac connective tissue cells; D. Very little staining is observed with anti-tropomyosin antibodies in these untreated control stem cells.

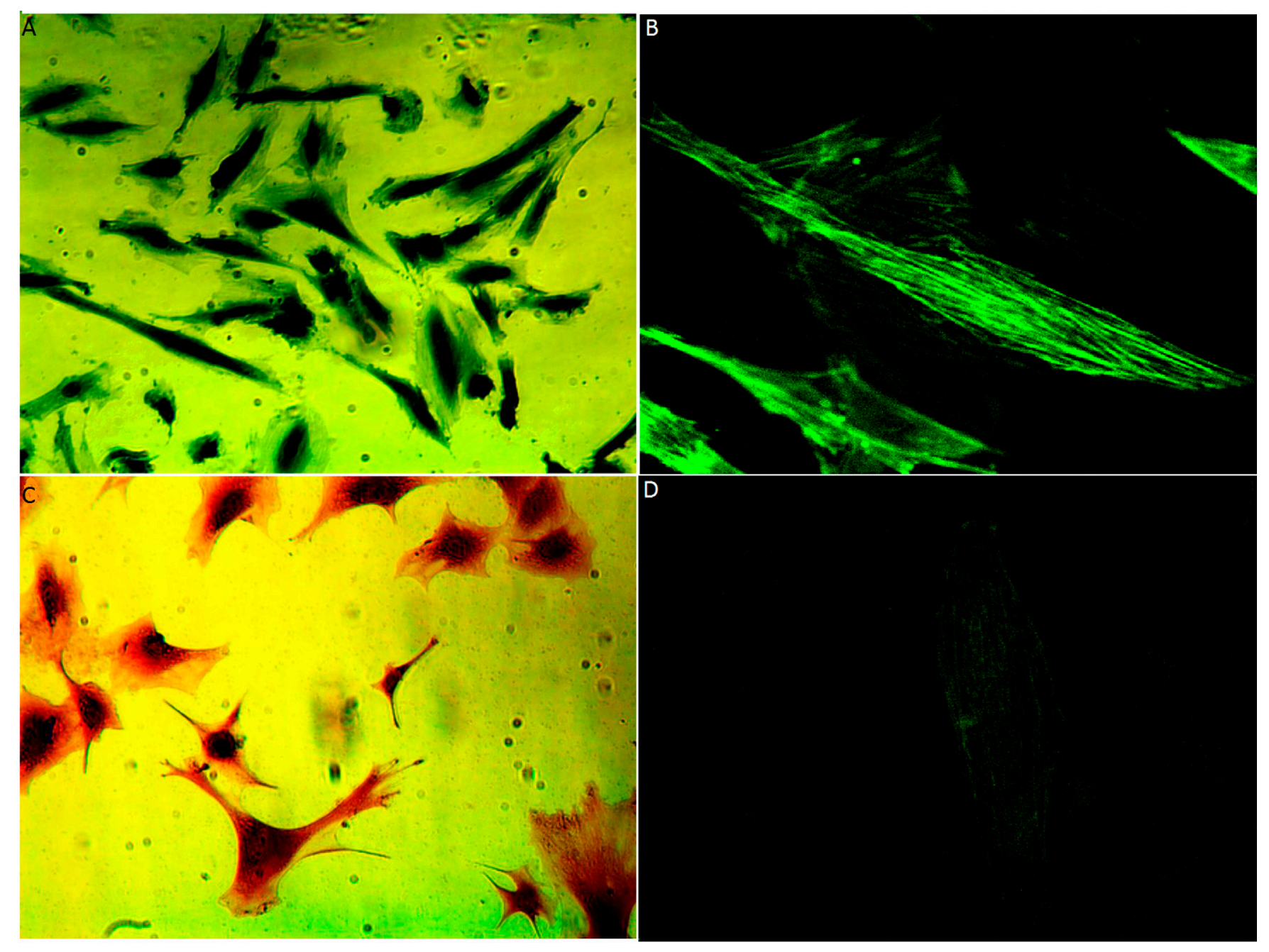

in $\mathrm{mESC}$-derived cardiomyocytes treated with miR499 (Fig. 4).

In our quantitative PCR studies, we examined the expression of various species of growth factors, Wnt, Sox and BmP, as well as the contractile proteins, troponin $\mathrm{T}$, tropomyosin and myosin, with and without miR-499c treatment. We found that expression of Wnt11 and Sox17 increased significantly in response to the miR-499c treatment (Fig. 5). Expression of other types of Wnt and Sox did not change significantly (not shown). Also with the expression of Wnt, cardiac marker contractile proteins including cardiac troponin T, tropomyosin and myosin increased significantly in 4 days and were at even higher levels after 8 days of the miR-499 treatment, corroborating the observations made using immunofluoresent confo- 
Figure 4: Fluorescent imaging of stem cell-derived cardiomyocytes treated with miR-499c after 8 days in culture stained with antibodies against: A. cardiac troponin T; B. tropomyosin; and C. $\alpha$-actinin. The miR-499c treated cells reveal significant staining for the muscle proteins showing they have differentiated into cardiomyocytes. Stem cells that are untreated, treated with lipofectin only or treated with RNA derived from a non-active clone show very little staining for these contractile cardiac muscle proteins.

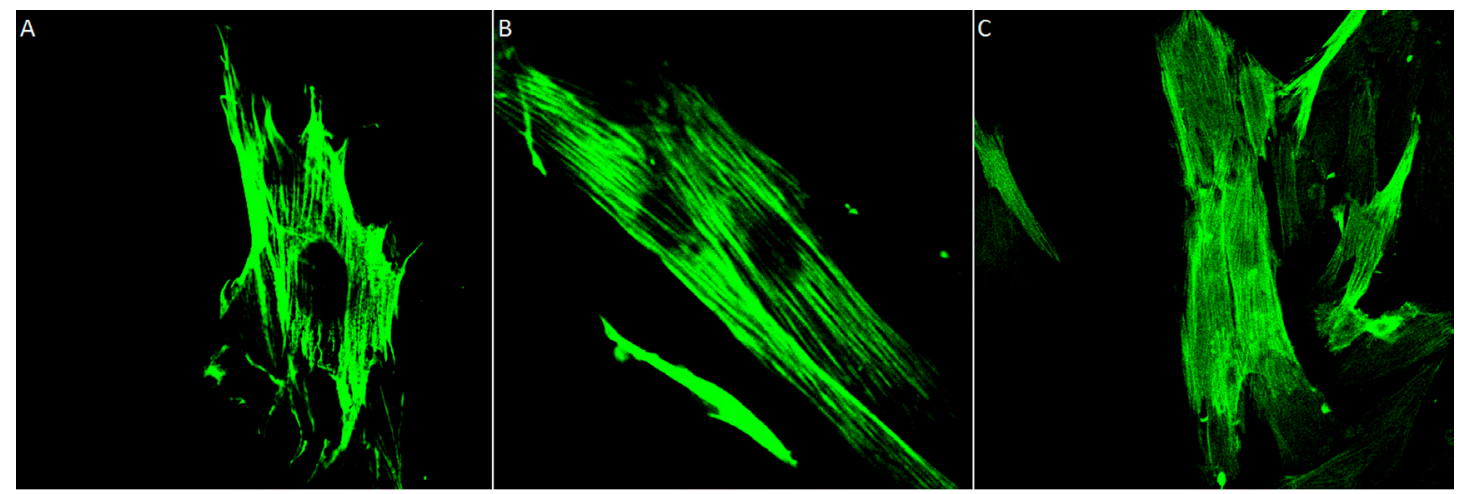

cal microscopy. Without miR-499c treatment, the Wnt11, Sox17, troponin T, tropomyosin and myosin did not increase significantly, but remained approximately at 0 day (base) levels (Fig. 5).

\section{Discussion}

In recent studies we have identified a new precursor of a microRNA which we have called miR-499c [2]. This novel miR-499c sequence partially overlaps with both miR-499a and miR-499b and contains an additional short 22 base pair (bp) sequence. In our previous publication, we showed that this microRNA promotes myofibrillogenesis and rescues mutant axolotl hearts by promoting the differentiation of non-muscle cells in the mutant myocardium into contracting cardiac muscle cells [2]. This fetal human heart microRNA appears to rescue the mutant axolotl hearts in a manner indistinguishable from our originally discovered axolotl myofibril inducing RNA (MIR) [5]. We hypothesize that these two RNAs promote myofibrillogenesis and rescue the mutant salamander hearts by binding and interacting with a common protein binding complex. They also in- duce differentiation of mouse embryonic stem cells. Spontaneous cardiomyocyte differentiation of mouse stem cells was described previously by Mummery et al. [6]. In that study, stem cells formed embryoid bodies (EBs) which then attached, proliferated and differentiated spontaneously. We found that spontaneous cardiomyocyte differentiation without RNA treatment was less than $10 \%$ and a large majority of these cells showed fibroblast-like shapes. Transfection of mouse ESCs with miR-499c significantly increased the formation of differentiated cardiomyocytes to $75-80 \%$ as revealed by immunohistochemical staining for cardiac specific troponin $T$, tropomyosin and $\alpha$-actinin and by directing the cell morphologies into typical cardiomyocyte forms (Fig. 4).

We also tested the ability of miR-499c to induce stem cell differentiation directly without the formation of EBs. We treated mouse embryonic stem cells daily for 6 hours with transfection medium containing miR499c. In this experiment, stem cells differentiated into definitive cardiomyocytes in 8 days as revealed by positive immunofluorescent staining for cardiac troponin $\mathrm{T}$ and tropomyosin and by showing typical myocardial cell morphologies. 
Figure 5: miR-499 induced expression of proteins in mouse ESCS at 0, 4 and 8 days. All of the proteins/ factors: Wnt11, Sox17, troponin T, tropomyosin and myosin show moderate increases after 4 days and more dramatic increases at 8 days in culture. In the control stem cells without miR499 treatment, even at 8 days in culture the proteins/factors remain essentially at the base levels recorded at 0 days.

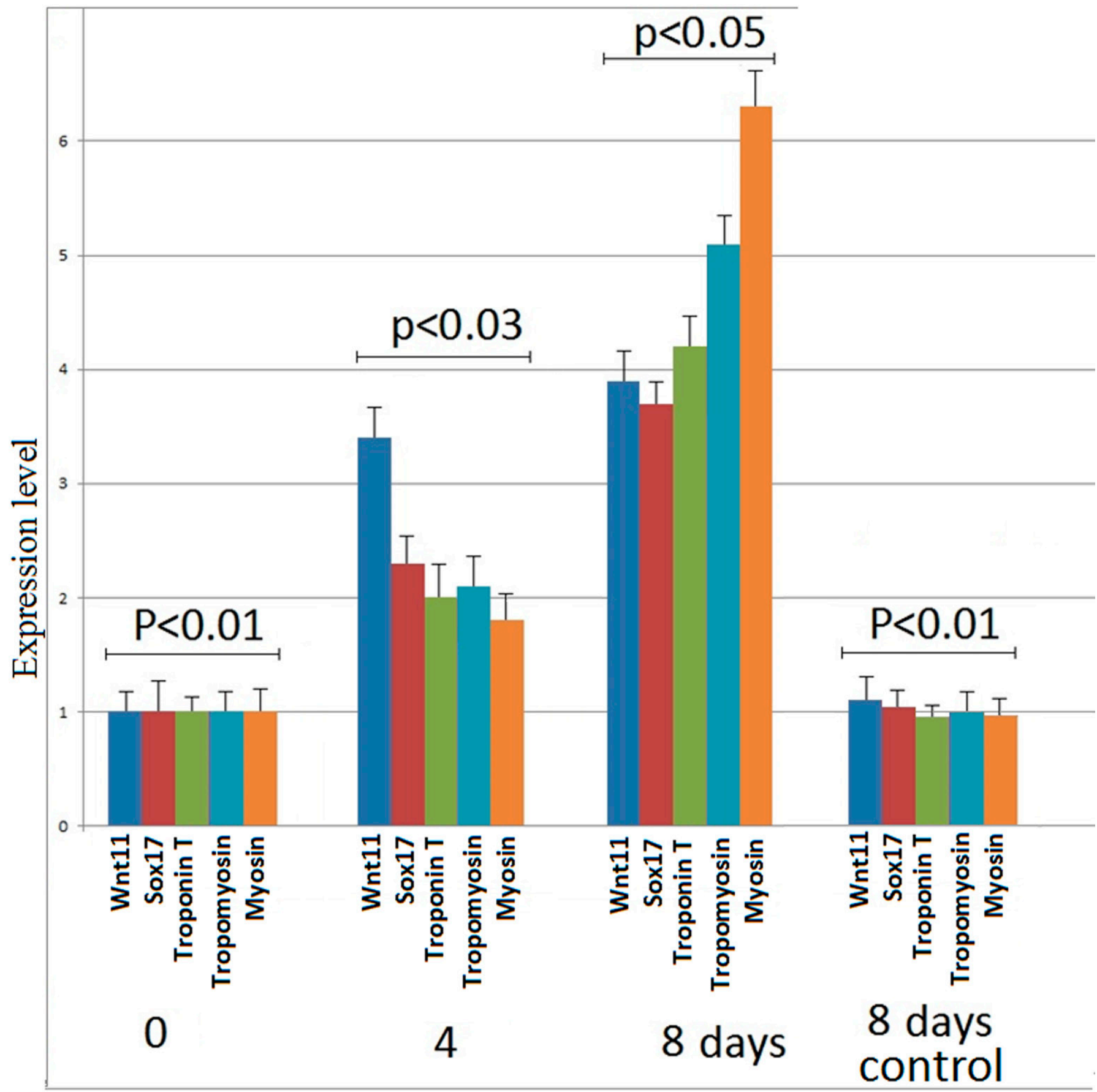


Interestingly, during normal in vivo embryonic heart development in mouse, the first cardiac muscle tissue also appears on the 8th day of development. Thus, our data have clearly and unequivocally demonstrated the ability of our newly-discovered miR499c to rescue mutant hearts in axolotl embryos by forming cardiomyocytes from nonmuscle cells and by inducing the differentiation of mouse embryonic stem cells to form into cardiomyocytes. Our findings are supported further by recent publications which show that miR-499 induces the differentiation of mesenchymal stem cells into cardiomyocytes [4]. In another research study, miR-499 transduction into human embryonic stem cells significantly increased the yield of human embryonic ventricular cardiomyocytes (from $48 \%$ to $72 \%$ ) along with increased contractile protein expression [7]. Further, it has been shown that miR-499 expression is significantly increased in differentiated cardiomyocytes [3].

Also, in our present study, expression of Sox6 did not show a statistically significant change with miR499c treatment, while expression of Sox11, which activates the non-canonical Wnt pathway, was significantly increased after 4 or 8 days. In addition, we found that Wnt11 expression correlated with an increase in expression of transcription factor Sox17. The research we report here, to our knowledge, is the first definitive evidence implicating Wnt11 as an important signal in cardiac differentiation. We hypothesize that Wnt11 may activate expression of the transcription factor, Sox17, which then initiates cardiac differentiation. Therefore, on the basis of our current results, one possible explanation is that in undifferentiated stem cells, transcription factors Oct 3/4 and Sox2 are expressed constitutively and bind to DNA to keep cells in a pluripotent condition, while in differentiating cells, Wnt11 activates expression of Sox17 and this in turn directs the cells to form into cardiomyocytes.

of the many individuals worldwide who suffer heart attacks each year, there are a number of treatments that currently are used to reduce their symptoms and help in the recovery process. Although these interventions improve the quality of life for some, many do not fully recover because connective scar tissue replaces the cardiac muscle in the infarcted area and the heart loses its efficiency in pumping the blood. The research we report here may lead to better treatments for patients in the future who suffer from myocardial infarctions or other heart diseases that negatively affect myocardial tissue. It is possible that the use of MiR-499c, in combination with induced pluripotent stem cells derived from that same patient, could be used to repair the damaged cardiac muscle tissue, leading to a much better prognosis for heart-attack victims in the future.

\section{Conclusion}

We have found that MiR-499c promotes the differentiation of mouse ESCs into cardiomyocytes as well as rescues the development of mutant embryonic hearts in the salamander, Ambystoma mexicanum. MiR-499c appears to be an important regulator of vertebrate heart embryonic development and cardiomyocyte differentiation. This may take place by Wnt11 activating the expression of Sox17 which then directs the cells to differentiate into cardiomyocytes. Our findings may have important future clinical implications for the treatment of human heart damaged from myocardial infarction or other disease processes by replacing the damaged tissue with stem cells derived from the patient and induced to form into new myocardial cells using the active microRNA, miR-499c. The hope would be that such patients, with their hearts repaired by newly-formed myocardial cells, could return to a normal lifestyle again.

\section{Competing interests}

The authors declare no competing interests. 


\section{Authors' contributions}

Dr. Lemanski served as the PI on the grants that funded the work. Dr. Lemanski and Dr. Kochegarov jointly designed the experiments and jointly wrote the manuscript. Ms Ashley Arms prepared the molecular clones from which the miR-499c was made. Dr. Michael Hanna worked with the molecular biology and qRT-PCR studies. We are grateful to Ms Sharon Lemanski for excellent secretarial support in the preparation of the manuscript.

\section{Acknowledgments}

This work is supported by an $\mathrm{NIH}$ grant (HL061246), American Heart Association grant (10GRNT4530001) and an NSF-RUI Award (1121151) to Larry F Lemanski and Michael Hanna.

\section{References}

1. Eulalio A, Mano M, Dal Ferro M, Zentilin L, Zacchigna S, Giacca M. Functional screening identifies miRNAs inducing cardiac regeneration. Nature 2012; 492: 376-381.

2. Kochegarov A, Moses A, Lian W, Hanna MC, Lemanski LF. A new unique form of microRNA from human heart, microRNA-499c, promotes myofibril formation and rescues cardiac development in mutant axolotl embryos. J Biomed Sci. 2013; 20: 1-20.

3. Hosoda T, Zheng H, Cabral-da-Silva M, Sanada F, Ide-Iwata N, Ogorek B, Ferreira-Martins J, Arranto C, D'Amario D, del Monte F, Urbanek F, D'Alessandro DA, Michler RE, Anversa P, Rota M, Kajstura J, Leri A. Human cardiac stem cell differentiation is regulated by a mircrine mechanism. Circ. 2011; 123: 12871296.

4. Zhang LL, Liu JJ, Liu F, Liu WH, Wang YS, Zhu B, Yu B. MiR-499 induces cardiac differentiation of rat mesenchymal stem cells through wnt/ $\beta$-catenin signaling pathway. Biochem Biophys Res Commun. 2012 Apr 20;420(4): 875-81. doi: 10.1016/j. bbrc.2012.03.092.

5. Zhang C, Jia P, Huang X, Sferrazza G, Athauda G, Achary MP, Wang J, Lemanski SL, Dube DK, Lemanski LF. Myofibrilinducing RNA (MIR) is essential for tropomyosin expression and myofibrillogenesis in axolotl hearts. J Biomed Sci. 2009; 16: 81.
6. Mummery C, Ward D, van den Brink C, Bird SD, Doevendans PA, Opthof T, Brutel de la Riviere A, Tertoolen L, van der Heyden M, Pera M. Cardiomyocyte differentiation of mouse and human embryonic stem cells. J Anat. 2002; 200: 233-242.

7. Fu JD, Rushing SN, Lieu DK, Chan CW, Kong CW, Geng L, Wilson KD, Chiamvimonvat N, Boheler KR, Wu JC, Keller G, Hajjar RJ, Li RA. Distinct roles of microRNA-1 and -499 in ventricular specification and functional maturation of human embryonic stem cell-derived cardiomyocytes. PLoS One. 2011; 6: e27417.

\section{Comment on this article:}

\section{(f) $[$ in $8+\mathbf{S} P$}

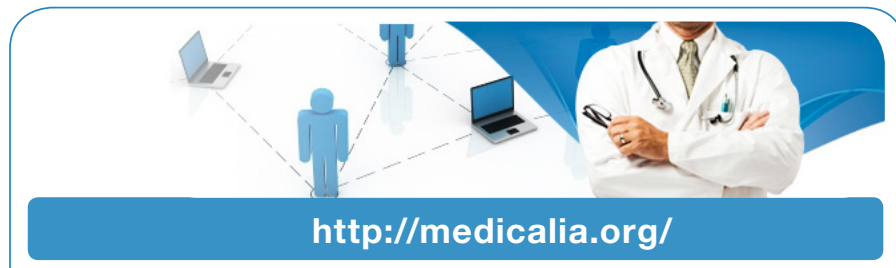

Where Doctors exchange clinical experiences, review their cases and share clinical knowledge. You can also access lots of medical publications for free. Join Now!

\section{Publish with iMedPub}

\section{http://www.imed.pub}

International Archives of Medicine is an open access journal publishing articles encompassing all aspects of medical science and clinical practice. IAM is considered a megajournal with independent sections on all areas of medicine. IAM is a really international journal with authors and board members from all around the world. The journal is widely indexed and classified Q1 in category Medicine. 